\title{
Structures postsuburbaines dans la région urbaine de Vienne
}

Post-suburban structures in the city region of Vienna

Post-suburbane Strukturen im Verdichtungsraum Wien

\section{Peter J. Görgl}

\section{(2) OpenEdition \\ Journals}

Édition électronique

URL : http://journals.openedition.org/rge/247

DOI : $10.4000 /$ rge. 247

ISSN : $2108-6478$

Éditeur

Association des géographes de l'Est

Édition imprimée

Date de publication : 1 juin 2005

Pagination : 133-144

ISSN : 0035-3213

Référence électronique

Peter J. Görgl, «Structures postsuburbaines dans la région urbaine de Vienne », Revue Géographique de l'Est [En ligne], vol. 45 / 3-4 | 2005, mis en ligne le 10 juin 2009, consulté le 08 septembre 2020. URL : http://journals.openedition.org/rge/247 ; DOI : https://doi.org/10.4000/rge.247

Ce document a été généré automatiquement le 8 septembre 2020

Tous droits réservés 


\title{
Structures postsuburbaines dans la région urbaine de Vienne
}

\author{
Post-suburban structures in the city region of Vienna \\ Post-suburbane Strukturen im Verdichtungsraum Wien
}

Peter J. Görgl

\section{NOTE DE L'ÉDITEUR}

Direction de la traduction : Gabriel Wackermann

\section{Introduction}

1 "Déclaration de guerre à Vienne », tel fut le titre donné par l'un des grands journaux quotidiens autrichiens, « Die Presse ", à son reportage sur le nombre croissant de zones d'activités modernes et sur l'aménagement d'infrastructures de transport dans l'espace densifié situé autour de la capitale fédérale («Die Presse», 20.6.05, p.1). Le départ d'entreprises et d'habitants, ainsi que l'apparition consécutive de rapports concurrentiels entre Vienne et les communes environnantes ne constituent pas (comme on peut le constater aussi dans toutes les grandes régions urbaines occidentales) un phénomène nouveau. Dès lors, toutefois, que l'on examine les tendances de cette évolution de plus près, il est possible de détecter, en matière qualitative, quelques spécificités qui apparaissent aussi comme très intéressantes, notamment dans l'optique "postsuburbia ", d'où l'intérêt de les observer de façon précise sous l'angle des sciences de l'espace. Comme le nombre de publications récentes sur la région urbaine de Vienne est encore facilement maîtrisable, force est de constater que le contexte théorique du phénomène " postsuburbia » n'est étudié qu'à titre marginal (p. ex. Mayer, 2004). La prise en compte de celui-ci est pourtant déterminant pour la compréhension des évolutions récentes de l'environnement spatial de Vienne. 
2 La présente contribution s'appuie sur trois petites analyses locales pour montrer comment cette mutation se réalise dans l'espace viennois et sur quels éléments les recherches à venir dans ce domaine peuvent s'appuyer concrètement. "Postsuburbia » a surgi de façon très différente dans chacun des trois cas, soit dans le cadre de nouvelles affectations fonctionnelles de friches industrielles, soit dans celui de la redécouverte de potentiels locaux préexistants, soit par l'affectation de terres agricoles à des fonctions urbaines quelque peu à l'écart des grands axes de communication.

\section{I. «Suburbia-post-suburbia » dans le contexte théorique}

3 Jusque vers la fin des années 1980, la suburbanisation "traditionnelle » a été l'expression de la relation dichotomique entre les noyaux urbains et leur espace environnant. Les villes ont connu une croissance qui a dépassé leurs limites administratives, alors qu'elles eurent entièrement à charge, fonctionnellement et spatialement, le développement des communes suburbaines (Brake, 2001, p. 16). Les entreprises, poussées par des besoins fonciers croissants et des prix moins élevés, la recherche d'une accessibilité plus adéquate, la mutation économique générale, le désir d'aller habiter au vert, pour échapper aux aspects négatifs de la ville (offre résidentielle insuffisante, trafic, criminalité,...), furent incitées à s'établir dans l'espace environnant. Le développement rapide de "suburbia» a été sous-tendue par la croissance démographique et l'amélioration du bien-être, ainsi que par la mise en œuvre d'une politique ayant beaucoup favorisé la propriété résidentielle individuelle (Einacker/ Mäding, 2004, p. 186). Ce processus fut déclenché par de jeunes familles à hauts revenus; elles ont acquis les maisons individuelles, les maisons jointives, ou les immeubles résidentiels collectifs, qui ont été construits le long des axes de liaison avec le centre. De nombreuses villes-dortoirs se sont formées autour des villes européennes ; elles sont caractérisées par une population résidentielle relativement homogène, vivant, dans bon nombre de cas, dans une ambiance architecturale impersonnelle. Le centre a conservé sa fonction en tant que lieu de localisation principale des activités de travail et d'approvisionnement; cependant, la croissance s'est effectuée essentiellement dans l'espace périphérique.

4 Considéré de cette manière, ce développement a conduit presque forcément, au cours du passé récent, à une mutation des rapports entre la ville-centre et «suburbia ». Au lieu de demeurer presque exclusivement focalisée autour du centre urbain, la région urbaine est de plus en plus organisée de façon polycentrique, car l'élargissement continu des infrastructures dans l'espace périphérique, dans l'optique d'une nouvelle logique économique, d'une modification des styles de vie et, par là même, d'un changement profond des préférences résidentielles, a conduit à la transformation de nombreuses communes en lieux attractifs. C'est ainsi qu'est apparue « Postsuburbia ».

Dans l'ensemble, la relation "post-suburbia/ville-centre » est toutefois caractérisée, moins par une mutation quantitative que qualitative. La région urbaine est formée par des implantations individuelles hautement spécialisées, reliées entre elles par un réseau de transport transversal à grand gabarit. Les différentes communes sont de plus en plus conscientes de leurs potentialités individuelles, de leurs atouts; elles n'hésitent pas à réaliser des concepts et des idées novatrices, dont certains requièrent des investissements financiers élevés. Les communes postsuburbaines s'émancipent ainsi 
de plus en plus de la ville-centre et se développent plus que jamais de manière autonome. Elles ont atteint une certaine "maturité » qui leur permet de se présenter comme des lieux attractifs, que ce soit dans le domaine du travail, du logement ou dans celui des loisirs. Ce processus permet, d'une part, à l'ensemble de la région urbaine de gagner en attractivité par rapport à d'autres régions ; il favorise aussi, d'autre part, la compétition intrarégionale entre les communes d'une agglomération urbaine.

6 Aring et Herfert évaluent ces tendances comme un processus d'urbanisation permanent de l'espace périphérique des villes-centres (Aring/Herfert, 2001, p. 43), un processus qui se manifeste dans un type d'urbanité éliminant le contraste classique entre villes et monde rural, tant au point de vue architectural qu'économique et socio-culturel (cf. Borsdorf, 2004, p. 12).

\section{La région urbaine de Vienne : peu de suburbanisation, beaucoup de postsuburbanisation}

7 Considérée du point de vue quantitatif, la suburbanisation de la région viennoise s'est effectuée et s'effectue toujours de façon modérée, tant en comparaison avec la situation autrichienne qu'en ce qui concerne le domaine international (tableau 1). L'évolution en cours permet de distinguer entre une phase très précoce de suburbanisation et une phase relativement récente. L'aire de peuplement le long de la «Südbahn » (mise en service en 1841) a déjà été urbanisée relativement tôt (cf.l'exemple suivant se rapportant à Mödling). Dès le milieu du XVIII ${ }^{e}$ siècle apparurent d'imposantes implantations industrielles, également le long de cet axe méridional, jusqu'à Wiener Neustadt, phénomène qui a conféré à cette région autrichienne, par suite de sa forte industrialisation, l'appellation de "Industrieviertel », une empreinte qui est demeurée en dépit de la mutation structurelle massive, intervenue entre-temps. Dans ce domaine, l'extension de la ville fut linéaire, non concentrique (Fassmann/Hatz 2004, p. 178 ss., fig. 1). 
Figure 1 : La région urbaine de Vienne, photo-satellite.

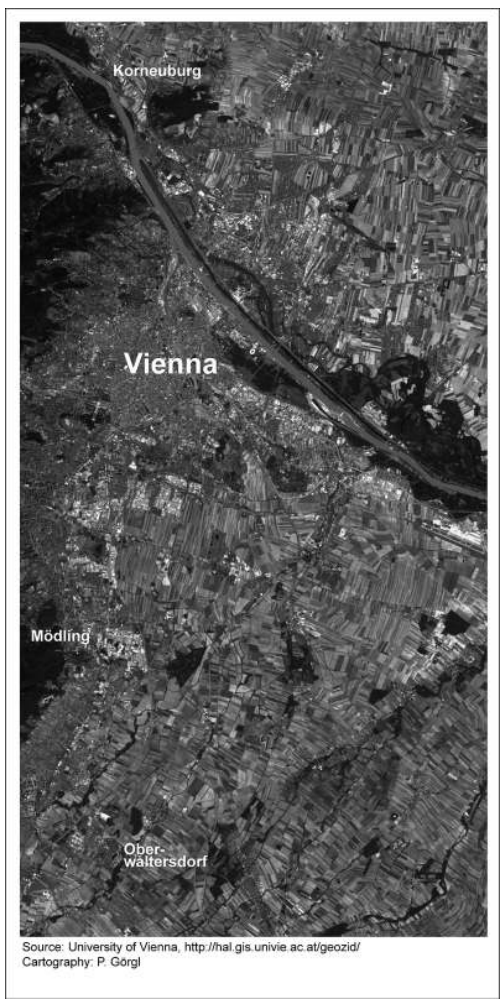

Tableau 1 : Migrations ville-espace aux alentours de Graz, Klagenfurt, Munich et Vienne.

\begin{tabular}{|c|c|c|c|c|}
\hline \multirow[t]{2}{*}{ Ville } & \multirow[t]{2}{*}{$\begin{array}{l}\text { Nombre d'habitants (recensement } \\
\text { 2001) }\end{array}$} & \multicolumn{2}{|c|}{$\begin{array}{l}\text { Solde migratoire annuel dans l'espace } \\
\text { périurbain }\end{array}$} & \multirow[t]{2}{*}{$\begin{array}{l}\text { Moyenne des } \\
\text { années }\end{array}$} \\
\hline & & chiffres abs & en $\%$ du nbre d'hab. & \\
\hline Graz & 226892 & $-925,6$ & $-0,41$ & $1996-2001$ \\
\hline Klagenfurt & 90275 & -135 & $-0,15$ & 1990-1999 \\
\hline Münich & 1336949 & -6.225 & $-0,48$ & $1996-2000$ \\
\hline Vienne & 1550123 & -3557 & $-0,23$ & $1996-2000$ \\
\hline
\end{tabular}

(Seules sont prises en compte les migrations dans l'espace situé dans le Land de Haute Autriche.) Source : BAUER-WOLF et al. (2003).

8 Alors que les villes allemandes ont connu un imposant mouvement migratoire villescampagnes dès le milieu des années 1960, la ville de Vienne n'a connu une dynamisation de ce processus qu'à partir de 1990. Quoique les communes directement attenantes à Vienne, notamment celles localisées le long de l'autoroute sud et de la "Südbahn", aient connu durant les décennies consécutives à la Seconde Guerre mondiale une croissance constante, la faible quantité de réalisations durant cette phase suburbaine s'explique en fin de compte par le fait que la ville de Vienne a disposé, à 
l'intérieur de ses limites administratives, de suffisamment de terrains à bâtir, pour satisfaire, en territoire urbain même, grâce à une politique de construction de logements innovante, la poussée démographique vers « l'extérieur ", qui a affecté aussi l'Autriche dans les années 1970: les taux de croissance dans les quartiers urbains extérieurs de Vienne furent à ce moment-là à deux chiffres (Bauer-Wolf et al. 2003, p. 1). Durant les dernières quinze années cependant, la propension à " habiter au vert " s'est affirmée plus concrètement : alors que la croissance démographique dans la ville de Vienne n'a atteint que $0,7 \%$ de 1991 à 2001, les communes périurbaines ont connu au total une progression de 6,7\%. Tandis que le développement s'est effectué d'abord le long des axes principaux de transport individuel et public, les aires situées entre les axes de croissance «traditionnels » ont gagné continuellement en attractivité au cours du passé récent, ce qui s'explique finalement par la faiblesse des prix du foncier et l'existence de réserves foncières suffisantes pour faire face à la pression résidentielle. Il faut remarquer que ce sont les circonscriptions périphériques septentrionales, demeurées encore des territoires d'émigration jusque dans les années 1980, qui ont gagné rapidement en attractivité et auxquelles on prédit la plus forte croissance (tableau 2).

Tableau 2 : Prévisions en nombre d'habitants

\begin{tabular}{|l||l|l|l|l|}
\hline \multirow{2}{*}{ Circonscription } & \multicolumn{4}{|c|}{ Nombre d'habitants, en chiffres absolus } \\
\cline { 2 - 6 } & 2001 & 2011 & 2021 & 2031 \\
\hline \hline Baden & 124865 & 132698 & 138627 & 142039 \\
\hline \hline Korneuburg & 67476 & 73133 & 77879 & 81398 \\
\hline Mödling & 104889 & 110854 & 115248 & 117348 \\
\hline Wien (Land fédéral) & 1527755 & 1589278 & 1631502 & 1637106 \\
\hline
\end{tabular}

Source : ÖROK-Prognosen 2001-2031, p. 128, conception : P. Görgl.

Dans la région urbaine de Vienne se sont constitués deux axes de croissance, de direction nord-sud et est-ouest, le long desquels sont situées les communes qui présentent les taux de croissance démographique les plus élevés. Ce sont surtout de jeunes familles au pouvoir d'achat élevé, qui ont quitté la ville-centre pour l'espace environnant; ce sont à présent avant tout des apports directs d'autres régions du pays ou de l'étranger, qui sont déterminants pour la croissance des communes de la région urbaine de Vienne (Fassmann/Hatz, 2004, p. 169).

Du point de vue de la morphologie urbaine, l'espace urbain densément bâti s'étend aujourd'hui de Wolkersdorf, au nord, jusqu'à Baden/Traiskirchen au sud (Fassmann/ Hatz, 2004, p. 166). En dépit de ce développement, et contrairement à ce qui se passe dans des régions urbaines comparables, telle celle de Munich, une réelle structure polycentrique continue à faire défaut dans l'espace viennois (Kagermeier et al., 2001). C'est ainsi que dans le domaine des localisations d'entreprises et du potentiel en surfaces de bureaux, la ville de Vienne, comparée à d'autres métropoles, est encore à 
même d'offrir des loyers modérés, ainsi qu'un bâti neuf, haut-de-gamme (Wienerberg City, Monte Laa, Millennium Tower, Donau City, Gasometer, etc.). Il convient en outre de signaler que la qualité de vie et un large éventail d'offres culturelles constituent des facteurs secondaires de localisation et contribuent à renforcer sensiblement l'attractivité de la ville.

11 Dans le cadre de cette relation, certaines communes périphériques gagnent néanmoins en importance. Le "Concorde Business Park» à Schwechat ou l'« Office Park Campus 21 ", qui jouxtent directement la limite de la ville, témoignent de l'importance grandissante de l'espace périurbain pour attirer de nouvelles entreprises. Leur accessibilité aisée et leur offre de loyers très avantageux, surtout pour les activités de production artisanale et industrielle, mais aussi pour les entreprises de services, sont particulièrement indiquées dès lors qu'un lieu d'implantation urbaine représentatif n'est pas indispensable. Même lorsque le développement, tel qu'il s'est manifesté dans la commune de «Wiener Neudorf » (création de plus de 10000 emplois pour à peine 8400 habitants), n'est pas nécessairement représentatif (Marik, 2002, p. 297), la concurrence sur le marché du travail entre la ville-centre et son espace périurbain constitue une nouvelle étape intéressante, dans la mesure où des deux côtés on peut mesurer ses atouts et sa dynamique ; cette situation est également incitative à la mise en œuvre de concepts novateurs (cf. l'exemple de Korneuburg).

12 La poursuite de l'aménagement ou de l'amélioration d'axes de communication déjà existants, en partie fortement soutenues par le Land de Basse-Autriche (telle que l'achèvement de la ceinture autoroutière autour de Vienne ou la construction de l'autoroute $\mathrm{A} 5$ dans le " Marchfeld », jusqu'à présent pas encore raccordé au réseau de communication de haut niveau, au nord-est de Vienne [cf. carte]) sont également une preuve d'attractivité croissante des communes périphériques. De la même façon, la compétition en matière de recherche d'implantations résidentielles et entrepreneuriales se poursuit entre les communes.

\section{Vers Postsuburbia I : Korneuburg - émancipation par rapport à la ville-centre par la restructuration}

13 Le district politique de Korneuburg fait partie des districts ayant la plus forte croissance dans la région de Vienne. Quoique les processus de suburbanisation se soient concentrés surtout dans les communes situées dans le sud-est, le district de Korneuburg a bénéficié, de 1991 à 2001, d'une croissance de $13 \%$; par là même, il s'est positionné nettement au-dessus de la moyenne régionale. Toutefois, cette phase de croissance est loin d'être achevée : la toute dernière prévision démographique de la conférence autrichienne del'aménagement du territoire (Österreichische Raumordnungskonferenz Örok) table sur une croissance d'environ 21 \% jusqu'en 2031 pour le district de Korneuburg, un taux qui place ce district en tête de l'ensemble des régions autrichiennes (Örok, 2005, p. 23).

14 Le chef-lieu du district de Korneuburg (12 478 habitants) est le centre administratif et le centre d'impulsion en matière d'aménagement de ce district politique, qui n'est situé qu'à sept kilomètres de la limite urbaine de Vienne et qui se trouve avantagé en matière d'accessibilité tant pour le trafic individuel (raccordement à l'autoroute A22 et à la route fédérale B3) que pour les transports publics (raccordement à la «Schnellbahn»). La conjonction de cette accessibilité avec les prix avantageux du 
foncier, dans le passé comme dans le présent, confère à ce district une avance sur l'espace périphérique méridional; elle offre des perspectives favorables au développement de la ville. Celle-ci est susceptible de devenir un important carrefour suprarégional de voies de communications, dans la mesure où il est prévu d'achever la rocade autoroutière de Vienne S1, et l'autoroute du Nord A5 (cf. Figure 2).

Figure 2 : Évolution démographique à Vienne et dans la région urbaine viennoise de 1991 à 2001.

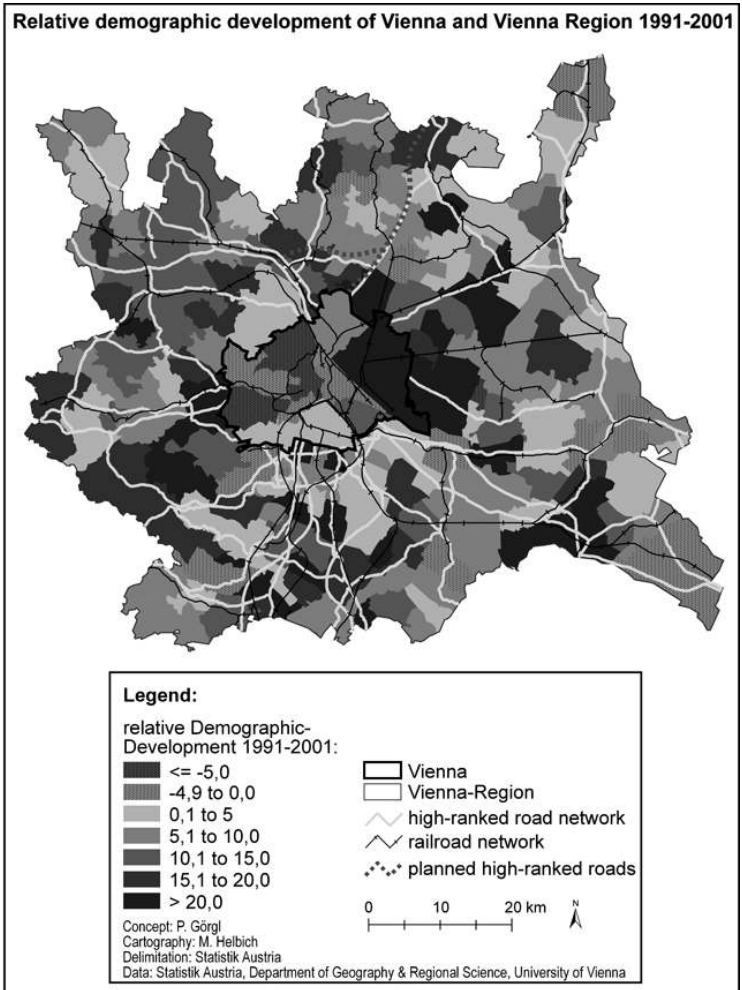

Le petit noyau historique de Korneuburg, une ville située sur la rive gauche du Danube, correspond dans beaucoup de domaines au stéréotype d'une commune suburbaine dite "typique ", marquée par de modestes maisons d'habitation de plain pied, construites au cours des dernières décennies, ainsi que par plusieurs lotissements hétérogènes de maisons monofamiliales, dont l'extension n'a été financée que par un minimum d'apports urbanistiques et architecturaux (Photo 1). L'évolution qui a conduit il y a quelques années à la transformation de l'ancien chantier naval de Korneuburg en un quartier urbain multifonctionnel révèle nettement que la commune ambitionne de rompre avec son vieil héritage industriel et son image historique pour se positionner en tant que centre tertiaire multifonctionnel moderne, fondé sur des activités de bureau et de services, ainsi que sur des offres culturelles et résidentielles. 
Photo 1 : Immeubles d'habitation à étages à Korneuburg.

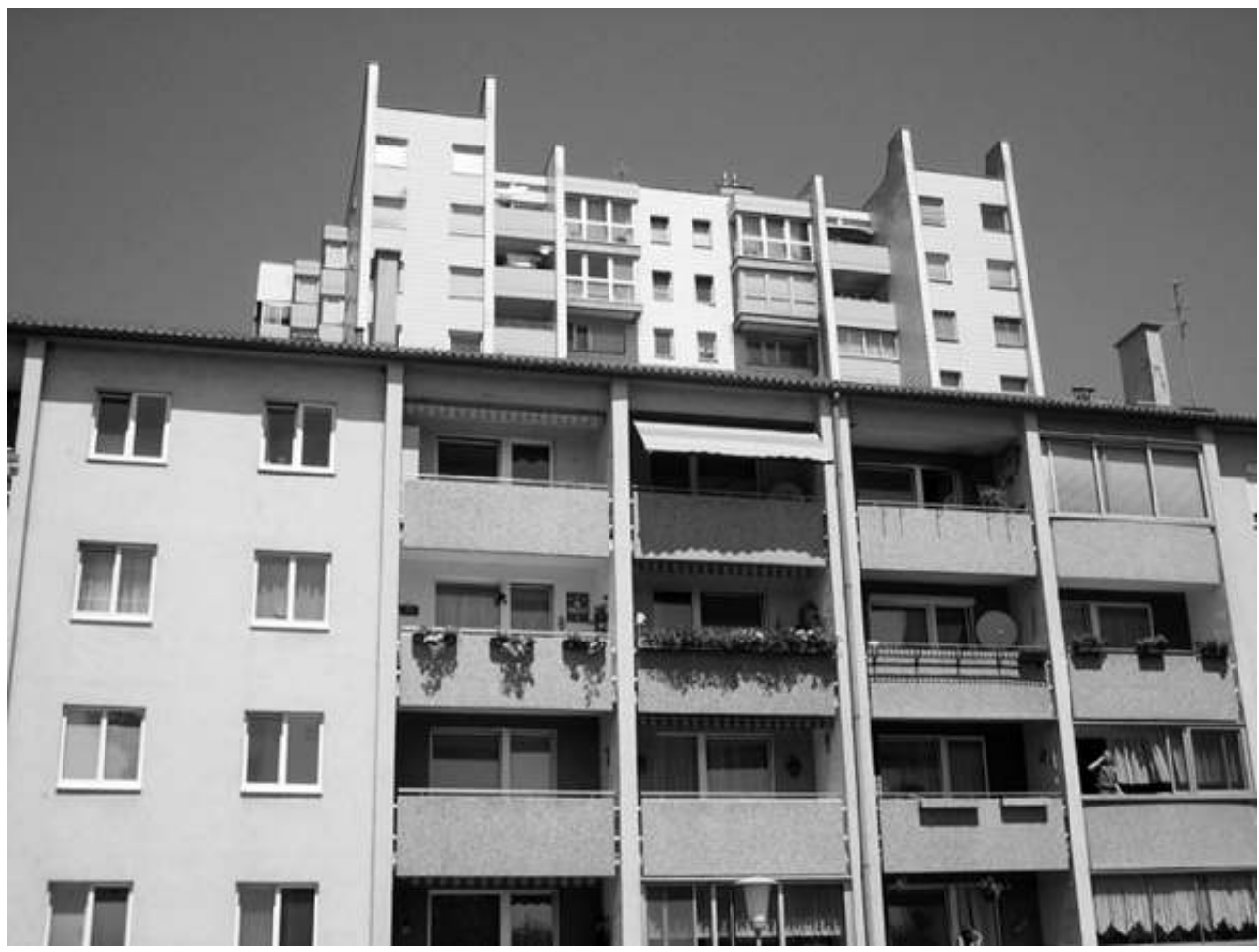

\section{A. Développement du chantier naval de Korneuburg et de l'espace environnant}

16 Jusqu'à son abandon définitif, au milieu des années 1990, le chantier naval de Korneuburg n'a pas été seulement d'une grande importance pour le chef-lieu du district, mais aussi une entreprise qui a contribué à conférer à celui-ci une forte identité, riche en traditions. Le chantier naval, dont l'histoire remonte à 1852, a été réaménagé à plusieurs reprises au cours des décennies passées. Durant les années 1960, à la demande de l'Union soviétique, Korneuburg a lancé plusieurs des bateaux de transport de passagers les plus modernes, de même que des bateaux de croisière, commandés par la société des bateaux à vapeur du Danube (Donaudampfschiffsfahrtgesellschaft DDSG), ainsi que des bateaux de pêche performants. En 1989, le chantier naval occupait 653 personnes. Parallèlement à de nombreux bateaux de transport de passagers, il s'y construisait aussi des grues et d'autres machines destinées au traitement du fret fluvial, à destination d'une clientèle internationale. Dans le cadre de la restructuration, la privatisation du chantier naval en 1991, fut vite suivie, en 1994, de la fermeture définitive de l'entreprise.

17 Le terrain souillé par les activités anciennes est demeuré une friche durant près de six années, lorsque l'administration publique se décida en l'an 2000 à le revitaliser par de grandes mesures de restructuration. A cet effet, furent créés un «WerftRevitalisierungs-GmbH (WRG) » et, par la ville, l'« Immofonds Korneuburg ». Ces deux entreprises acquirent chacune la moitié du terrain; le domaine d'activité de l'Immofonds n'était pas limité au terrain propre du nouveau chantier naval, mais 
étendu à la coordination des potentialités, de l'analyse des besoins et des mesures de développement de l'ensemble du territoire urbain.

\section{B. Le nouveau concept de la fonctionnalité des terrains - Vers la «Postsuburbia »?}

La réhabilitation complète et onéreuse de la friche navale, sur une superficie de plus de $60000 \mathrm{~m}^{2}$, a constitué le point de départ indispensable de la réalisation des projets ambitieux signalés ci-dessus. Elle va s'achever dans les mois à venir, après plusieurs années de réalisation. La revitalisation et le changement d'affectation de cette aire en font à présent un point d'ancrage du développement de l'ensemble de la commune de Neuerburg, conformément au nouveau concept d'aménagement, adopté en 2000. Celuici prévoit la création d'une zone de loisirs, d'activités et d'habitation, dans laquelle l'intégration portuaire et celle d'autres fonctions relatives aux usages de l'eau doivent promouvoir l'environnement, notamment celui constitué autour du Danube. Il s'agit en somme d'un concept multifonctionnel, dont la réalisation doit susciter un nouveau quartier urbain de plus de 2000 habitants et avec plus de 1000 emplois hautement qualifiés (photo 2).

Photo 2 : Vue aérienne du chantier naval de Korneuburg, projets prévus et réalisés.

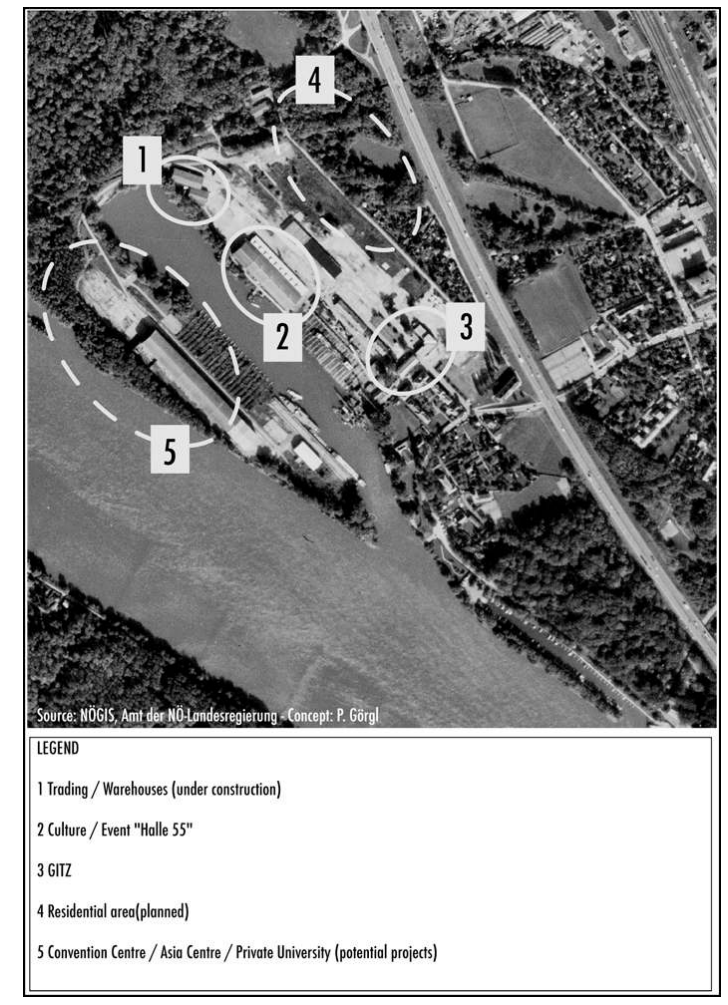

19 Le développement amorcé sous la dénomination très médiatique, à résonances internationales, de «Blue Danube Park », de l'ancien terrain du chantier naval repose sur un " concept à quatre colonnes", en vue de rendre cette aire attractive pour une palette d'investisseurs aussi large que possible : 


\section{Le « Gründer-, Innovations- und Technologiezentrum GITZ »}

20 L'existant déjà rénové et le projet de construction d'un immeuble permettent de disposer de $6000 \mathrm{~m}^{2}$, auxquels il faut ajouter $5000 \mathrm{~m}^{2}$ de surfaces de bureaux et d'entreprises, ainsi que des halls de stockage, qui sont utilisés à présent par 45 firmes. Le «Gründerzentrum » propose des aides financières pour le lancement de jeunes entreprises et met les infrastructures indispensables à la disposition des firmes.

\section{Le centre administratif international}

21 Initialement, l'ancien immeuble « Halle 55 » du vieux chantier naval n'était conçu que comme un lieu provisoire destiné à l'organisation de manifestations. A la fin de l'année 2004, il a cependant été transformé en lieu de rencontre international multifonctionnel, disposant d'une scène aquatique, avec le soutien du gouvernement du Land de Basse-Autriche. Ce lieu est à même d'accueillir jusqu'à 900 visiteurs lors de manifestations et d'événements divers; il est ainsi sollicité par un important festival interrégional de musique et de culture, ce qui a permis à la ville de Korneuburg de bénéficier d'une forte attractivité, notamment chez les jeunes. A présent, on y organise chaque année, 60 à 65 manifestations. La construction d'un second hall d'accueil est déjà programmée ; l'offre gastronomique et des infrastructures complémentaires sont également lancées.

\section{Sport, loisirs et détente}

Les structures ultra-modernes d'un Yachtclub sont en train de naître sur une surface d'environ $4000 \mathrm{~m}^{2} \mathrm{du}$ "Blue Danube Park». Au terme de la première phase d'aménagement, elles comporteront un imposant "Clubhouse ", une » Sliparea » et environ 60 anneaux pour yachts privés. Lors de la seconde phase, il est prévu une extension des infrastructures en vue de l'accueil de 140 anneaux supplémentaires.

\section{Préparation de la mise en valeur de la presqu'île par un projet à vocation internationale}

Un investisseur étranger envisage de construire un "Asia-Center » sur la presqu'île très pittoresque du Danube et de ses abords verdoyants. Ce centre comportera, dans une première phase, un hôtel quatre étoiles d'une centaine de lits, avec des salles de réunion, le tout complété par une gastronomie thématique chinoise de haut niveau, ainsi que par un «Asia Spa ». Au cours d'une seconde phase il est prévu de réaliser un «Business Center» à la chinoise avec des salles de représentation spécialement conçues pour des firmes et des représentants des autorités chinoises.

24 Ce cadre doit être parachevé par des formules alternatives et complémentaires (Büro Paula, 2002) destinées à accompagner la création, sur la presqu'île, d'une université privée.

25 Il est prévu également de réaliser un second accostage pour bateaux dans le cadre du chantier naval, en vue d'associer plus efficacement les multiples offres gastronomiques, culturelles et récréatives avec le tourisme fluvial.

L'office de management général de la société de développement foncier, qui est à la base de ce programme, est confiante dans les chances de réalisation des divers projets 
au cours de la décennie à venir, d'autant plus que dans tous les domaines envisagés les premières discussions avec les investisseurs et les partenaires intéressés ont donné des résultats positifs; de plus, des documents concrets et l'élaboration de mesures de construction ont déjà été finalisés. En ce qui concerne les projets de construction, le GITZ est désormais entièrement bouclé.

La réussite du réaménagement de cette partie de la ville dépendra aussi de la façon dont ce quartier sera fonctionnellement intégré aux infrastructures urbaines existantes. Jusqu'à présent, l'autoroute A22 forme surtout une barrière urbanistique et éventuellement mentale, bien que la gare ferroviaire soit seulement à environ dix minutes à pied et le centre-ville à un quart-d'heure de marche.

Pour le moment, des négociations sont en cours, à l'échelle du Land, en ce qui concerne le raccordement direct à l'A22, du domaine du «Blue Danube Park». Quoique le nouveau quartier ne puisse pas être rapproché du noyau urbain existant, la réussite de cette opération pourrait améliorer l'accessibilité et l'attractivité suprarégionale.

\section{Une nouvelle concurrence pour Vienne?}

$\mathrm{Si}$, dans le processus classique de suburbanisation, le transfert de fonctions depuis le noyau urbain vers l'espace périphérique est devenu classique, le développement au cours des dernières années de Korneuburg, illustre de façon saisissante les tendances postsuburbaines. Il est évident que pour cette évolution la proximité de la métropole internationale de Vienne est déterminante. Toutefois, dans la mesure où, à Korneuburg, l'accent est mis sur une offre propre, hautement attractive et diversifiée, en potentialités d'implantation d'entreprises, de rencontres, d'offres résidentielles, d'événements culturels et d'excursions, etc., la dépendance par rapport à la "grande ville» est atténuée. Korneuburg se présente plutôt comme un petit concurrent bien équipé, apte à être attractif par ses réserves foncières à prix modéré et par ses structures flexibles, de taille modeste. Dès lors que les aménagements du réseau interrégional de transport, évoqués ci-dessus, auront été réalisés, et que les projets relatifs au «Blue Danube Park " pourront être achevés dans leur ensemble, la ville de Korneuburg pourra jouer un rôle important comme "carrefour primaire " dans la région urbaine viennoise. Les bases indispensables à cette réussite sont d'ores et déjà en place.

\section{En route pour Postsuburbia II : Mödling arrachée au sommeil de la Belle au bois dormant pour devenir une commune « lifestyle »}

Les localités situées sur les versants méridionaux du Wienerwald ont vécu à partir du milieu du XVIII ${ }^{e}$ siècle un énorme développement, parce qu'elles ont été découvertes comme des lieux récréatifs de proximité par la noblesse urbaine et des artistes réputés dans un premier temps, puis, très vite après, aussi par la bourgeoisie viennoise, dès lors que l'ouverture de l'axe ferroviaire sud, en 1841, en a facilité et accéléré l'accessibilité. Par là même, une première phase précoce de suburbanisation a été rendue possible. C'est ici que se sont développés jusqu'à la Grande guerre et la fin de la Monarchie des sites viticoles pittoresques, tels que Perchtoldsdorf, Mödling ou Baden, devenus pour 
une large clientèle des villégiatures structurées par des thermes, salons de cure thermale et villas de la Belle époque (photo 3).

Photo 3 : Bâti ancien, rénové à Mödling.

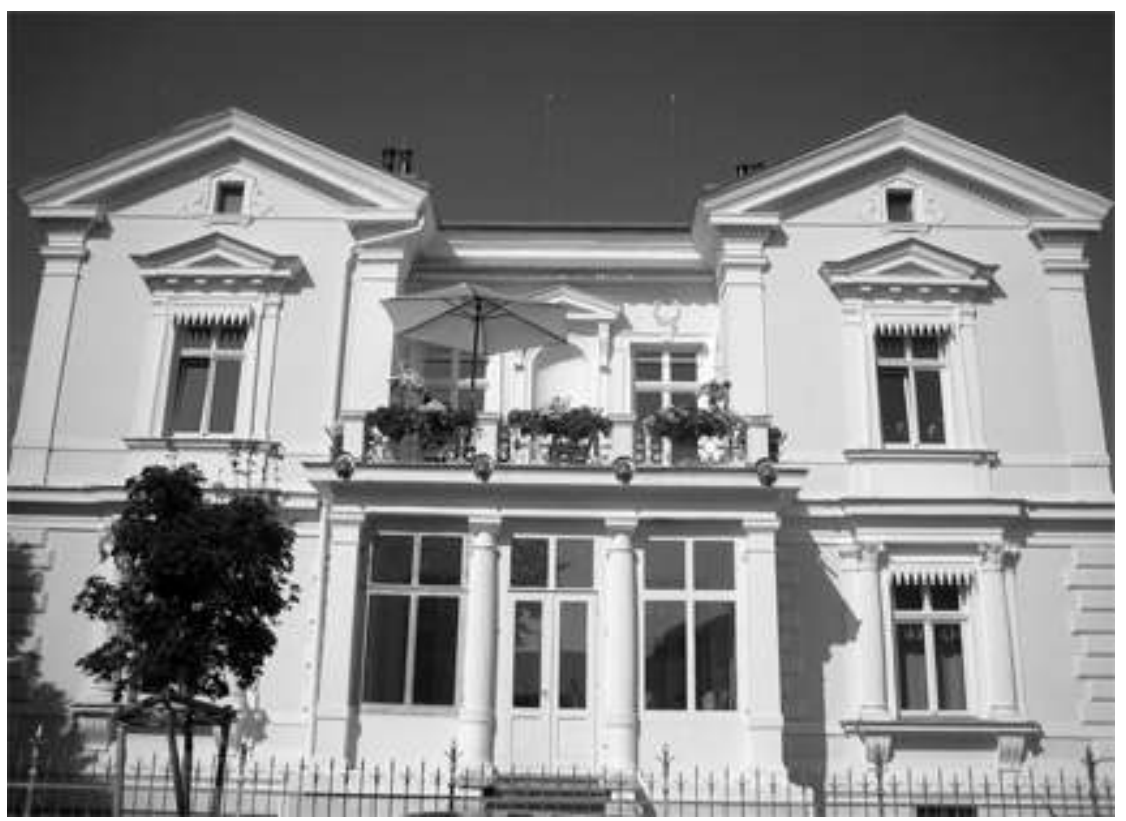

Le chef-lieu actuel de district, Mödling, était particulièrement apprécié par des artistes et intellectuels; la vieille Burg de Mödling a été rénovée; sur le revers de quelques versants du Wienerwald, le prince Jean I. de Liechtenstein, s'appuyant sur des ruines et temples artificiels préexistants, a tenté d'adapter le paysage autour de Mödling aux projections romantiques historiques. La ville a connu simultanément un imposant mouvement d'industrialisation. Dans sa partie orientale sont nées de nombreuses fabriques relevant de l'industrie lourde; quelques petits lotissements ouvriers demeurent encore aujourd'hui des témoins de cette période de l'histoire urbaine. Cette évolution a été accompagnée d'une forte croissance démographique : alors que Mödling ne comptait en 1811 que 2000 habitants, elle en avait déjà 18000 à peine un siècle après (23 288 actuellement). Cette croissance rapide, qui s'est répercutée sur l'aménagement d'une infrastructure moderne (hôpital, réseau de canaux, académie militaire, écoles,...), a été brutalement interrompue durant la Première Guerre mondiale; même la courte période de renouveau, propre à l'Entre-deux-guerres, n'a pas pu empêcher que la ville ne tombe dans une sorte de sommeil de la belle au bois dormant ; ce n'est qu'au cours des dernières années qu'elle s'est à nouveau réveillée.

Sérieusement endommagée durant la Seconde Guerre mondiale, condamnée à une stagnation presque complète pendant les années suivantes, sous l'occupation soviétique (jusqu'en 1955), la ville a dû attendre le départ des troupes pour connaître une reconstruction efficace : 3000 appartements nouveaux furent construits jusqu'en 1975, surtout durant l'époque caractérisée par des immeubles à étages. Quoique la pittoresque vieille ville ait été dans l'ensemble préservée et que le prix du foncier, ainsi que celui de l'immobilier soient demeurés très faibles, cette localité n'a pas pu renouer avec son passé prestigieux du temps de la Monarchie. Elle a été constamment victime de la démesure du "strip development» le long de son axe méridional. Mödling se situe donc aujourd'hui dans le couloir bâti de manière continue entre la limite urbaine 
méridionale de Vienne et Baden/Traiskirchen. Depuis environ une décennie, il est toutefois possible d'observer un net accroissement (qualitatif) et l'émergence de nouveaux équipements, qui permettent d'affirmer légitimement que la ville s'oriente délibérément, à l'appui de ses propres potentialités, vers un type de commune " lifestyle ", bien que des études empiriques fassent encore défaut à ce sujet. Mayer a perçu dans le vieux bâti bien conservé de communes telles que celles de Baden ou de Mödling un potentiel de forte identification (Mayer, 2004, p.99), qui joue non seulement un rôle important pour la population en place, mais aussi pour celle à venir. Nous soutenons la thèse selon laquelle ce phénomène est l'expression d'un engagement très fort en faveur du développement urbain et de l'identification prononcée entre le citoyen et son lieu de résidence, évolution qui se traduit par l'existence de nombreuses initiatives de citoyens, d'actions en faveur du marketing urbain, d'esquisses de développement urbain, etc., contrastant nettement avec le stéréotype de la «villedortoir suburbaine ».

Dans le cas concret de Mödling, ce processus semble être porté avant tout par des habitants disposant de moyens appropriés, soit parmi les nouveaux arrivants, soit appartenant essentiellement à la seconde génération des résidents en aire suburbaine : il s'agit donc des enfants arrivés à l'âge professionnel, issus des familles à niveau de formation et à revenu relativement élevés, qui se sont implantés dans le sud de Vienne durant les années 1970. Par leur "passé spécifique ", ils sont liés fortement à leur lieu résidentiel. L'administration locale a mis à profit cette situation favorable par le développement d'un concept approprié de marketing urbain, très discuté. officiellement et indiscutablement, la ville essaie de conforter sa position dans la compétition intra-régionale (cf. www.stadtmarketing-moedling.at et www.moedling.at).

La prédominance de groupes d'habitants s'identifiant à la ville et disposant de revenus élevés, tant à Mödling que dans le district de ce chef-lieu (index de pouvoir d'achat de Mödling: 134,5 points, contre 102,3 points en Basse-Autriche; voir www.stadtmarketing-moedling.at/kaufkraft.html), se manifeste aussi dans la structure du commerce de détail de la ville. L'image du centre ville est sous-tendue par la présence de filiales de chaînes d'habillement et alimentaires de haut niveau, de même que par celle de "niches", telles que des galeries, des boutiques de mode, des entreprises d'équipement et de restauration, des antiquaires, des vinothèques, activités complétées par des entreprises de bien-être sanitaire, de type « wellness », de médecine alternative, des centres de méditation, sans oublier la gastronomie internationale de haut niveau.

Cette offre de structure diversifiée, orientée vers la satisfaction des besoins d'un style de vie alternatif coûteux, repose sur une clientèle fortunée, qui s'identifie étroitement avec la ville et qui, selon Kulke, fait partie de cette clientèle très mobile à flexibilité spatiale élevée en matière commerciale (Kulke, 2002, p. 58). Dans le cas d'une identification moindre avec le lieu d'habitation, la demande d'une telle offre nécessiterait la présence de ce centre ville à une distance maximale d'une demi-heure.

Möding peut servir d'exemple d'un processus de suburbanisation qui a conduit, après deux décennies, l'administration municipale à prendre conscience des nouvelles potentialités de la ville, c'est-à-dire d'une structure urbaine et d'un bâti représentatif approprié. Compte tenu des réserves foncières limitées, les autorités de la ville ont mis l'accent sur la rénovation qualitative qui se traduit par des opérations de 
restructuration et d'assainissement autant que par le marketing urbain et la discussion ouverte sur le façonnement de l'image urbaine. Ce principe est également défendu par les résidents à revenus élevés et à fort attachement identitaire, soucieux de réaliser leurs aspirations individuelles visant un style de vie de haut niveau. C'est ainsi que Mödling est devenu un centre régional qui privilégie en premier lieu une clientèle poursuivant des projets individuels et non pas des finalités proprement « urbaines ». La réalisation de telles conceptions de styles de vie (postmodernes) n'est possible que dans les localités marquées par la postsuburbanisation (Borsdorf, 2004, p. 12), dont Mödling constitue une illustration impressionnante.

\section{Vers «Postsuburbia » : « Fontana », une résidence au vert pour classes sociales aisées}

À environ $20 \mathrm{~km}$ au sud de Vienne, un entrepreneur austro-canadien a construit en 1994 le «Fontana-Park", qui occupe une position spécifique non seulement dans la région de Vienne, mais même à l'échelle européenne, tant en ce qui concerne sa mixité fonctionnelle que les concepts urbanistiques et architecturaux originaux qui s'y sont développés ${ }^{1}$.

Lors de la création, il y a plus d'une décennie, de la filiale européenne de sa firme, l'investisseur unique a souhaité combiner trois fonctions en un concept esthétique unique : la fonction professionnelle, la fonction résidentielle et la fonction récréative. Deux autres critères devaient être retenus pour le choix du lieu d'implantation de ce projet : un cadre historique attractif et une bonne accessibilité en matière de voies de communication. D'emblée il ne pouvait être question que d'une localisation dans l'espace périphérique méridional de la ville de Vienne, notamment en raison de l'attractivité paysagère du "Wienerwald» et de la proximité de communes offrant «traditionnellement » des résidences de haut niveau (Baden, Mödling, Perchtoldsdorf). Un domaine agricole de 17 hectares, comportant un vieux château a été acquis à la périphérie de la commune d'oberwaltersdorf, et a servi de point de départ à la réalisation de "Fontana ». Le concept, qui ressemblait à un parc, tranchait nettement, dès le début, avec l'espace environnant, dans la mesure où il était l'expression des aspirations des couches sociales aisées voire très aisées, en ce qui concerne l'espace résidentiel et récréatif. Cette orientation a conduit à la réalisation d'un lac pour la baignade, d'un terrain de golf à 18 trous, de dimension européenne, ainsi qu'à la construction d'un "Clubhouse " (photo 4) avec restaurant haut de gamme, tennis à la fois " out " et «indoor ", et "fitnessclub ». C'est dans une ambiance très "soignée ", créée à grand renfort de moyens financiers, que furent construits, en deux phases, d'abord 80 maisons individuelles, puis 110 appartements (photo 5). La centrale de la firme emploie environ 140 personnes, dont 60 ingénieurs dans la division R\&D. Le château rénové d'oberwaltersdorf est le lieu de résidence du fondateur de la firme. 
Photo 4 : Fontana - Clubhouse.

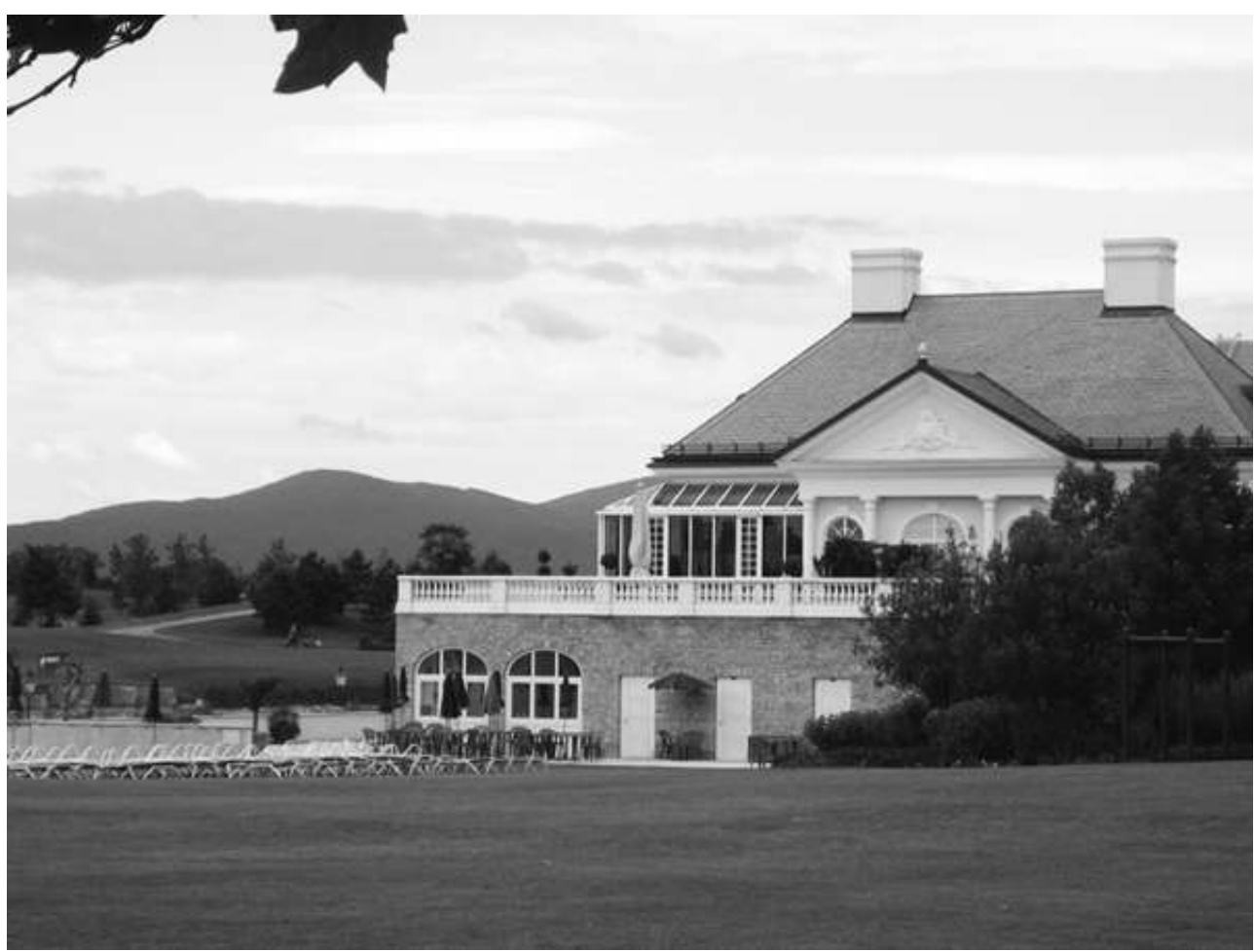

Photo 5 : Appartements de standing à Fontana.

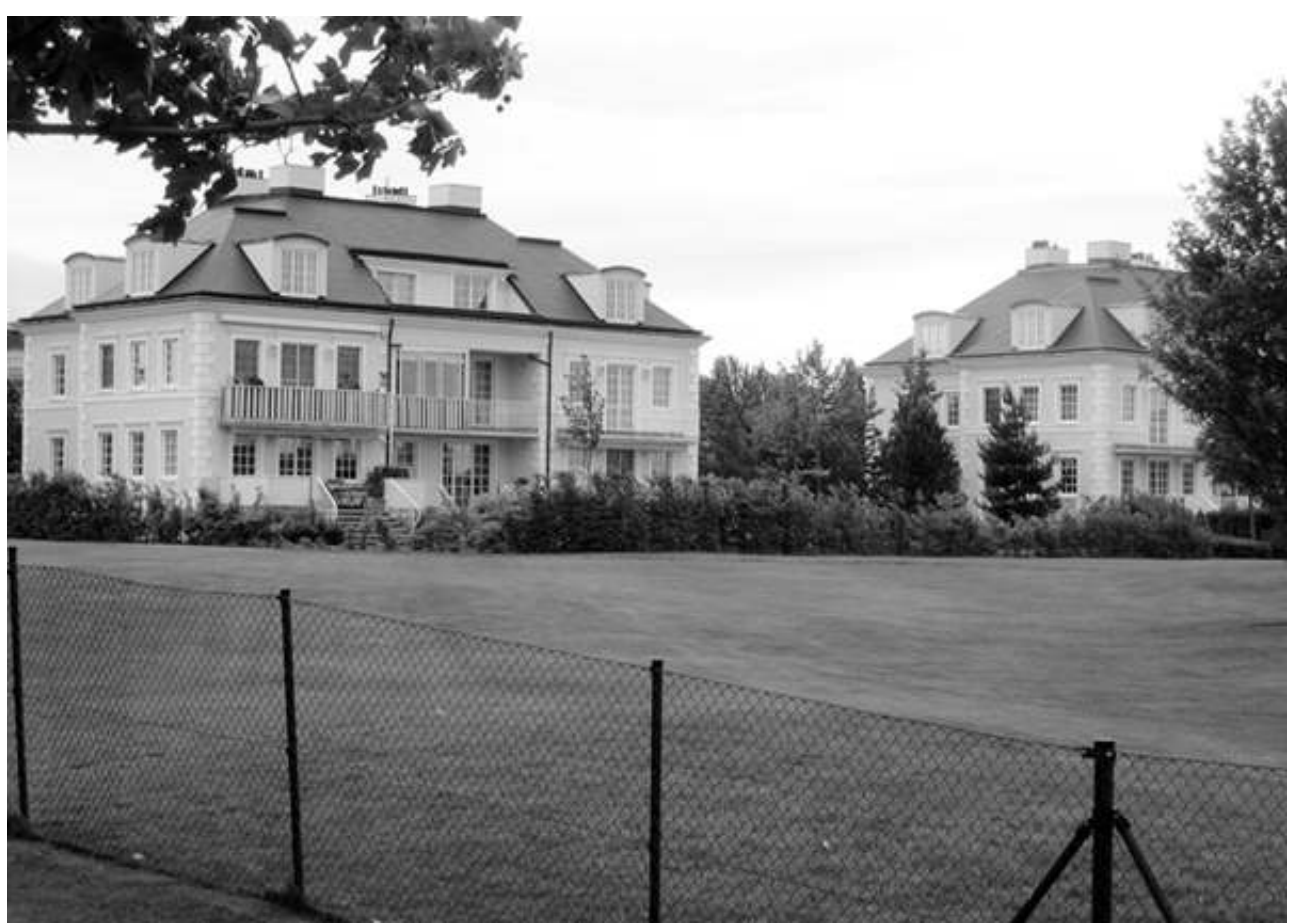

L'ensemble de l'implantation se distingue nettement de l'espace environnant; il ne comporte qu'une voie d'accès principale et est entièrement clôturé ; le terrain de golf est rendu invisible depuis l'extérieur par un glacis gazonné ; il n'est accessible qu'à partir de l'arrière par une petite route. Le domaine tout entier est aménagé dans un cadre d'aspect homogène; maisons et façades répondent à un principe architectural 
unique (photo 6). Toutefois, il ne s'agit pas de constructions conformes à un catalogue. Tout le restant - cadre général, conception de l'ensemble, ... - correspond aux désirs individuels des clients.

Photo 6 : Rue résidentielle à maisons individuelles à Fontana.

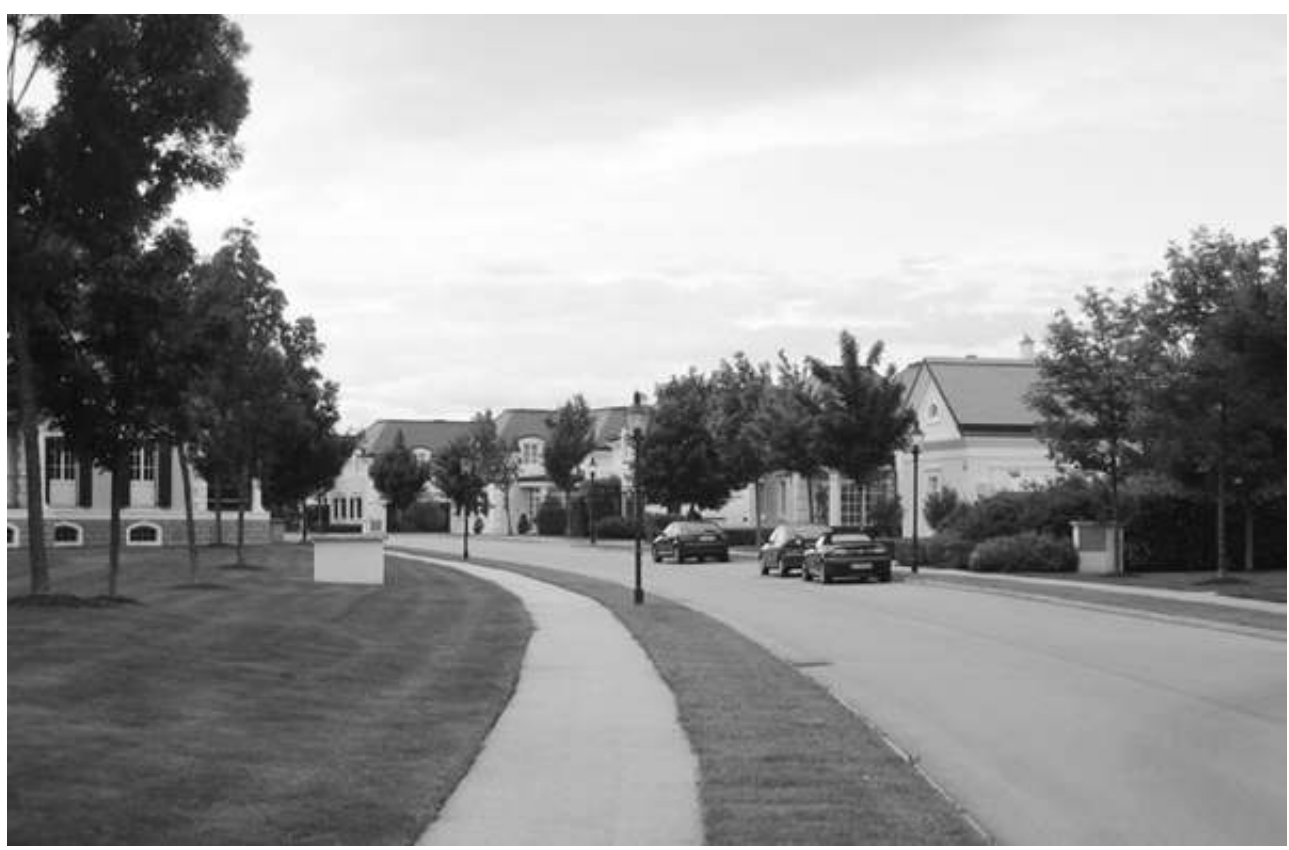

Par contraste avec le début de la première phase de construction, la société chargée de l'aménagement et du développement de «Fontana ", n'assume plus le rôle de maître d'ouvrage; elle se limite à la vente des parcelles, ainsi qu'à l'entretien des espaces verts, pour lequel chaque ménage paie un forfait annuel versé au Fonds de conservation Fontana ("Fontana Erhaltungsfonds»), lié à un cahier des charges. L'utilisation du lac pour la baignade est soumise à redevance, de même que celle du terrain de golf et des autres équipements.

\section{A. Fontana, une « lifestyle community »?}

41 L'offre haut-de-gamme en matières récréatives et sportives montre qu'il s'agit là d'une communauté typiquement "lifestyle». Des entretiens réalisés avec des résidents incitent cependant à la prudence. Il est vrai que les équipements sportifs sont fréquentés et que la pratique du golf joue un rôle important de détente quotidienne, après la journée de travail, pour de nombreux habitants. Il convient néanmoins de s'interroger sur l'origine des résidents de Fontana et sur les motivations premières en ce qui concerne le fait d'aller habiter Fontana.

La ségrégation est déjà évidente par suite des prix élevés du foncier, comparables avec ceux des niveaux les plus hauts observés à Vienne même. C'est pourquoi la plupart des résidents sont soit des cadres supérieurs d'entreprises travaillant dans le secteur élevé ou très élevé du marketing, soit des chefs d'entreprise indépendants ou des propriétaires de firmes, des avocats, médecins, ... Seuls quelques-uns d'entre eux travaillent sur place, dans la filiale européenne de la société de promotion immobilière. 
Le nombre de jeunes ménages qui se sont décidés à opter pour Fontana est surprenant ; le choix opéré correspond visiblement à des projets de vie personnels.

Fontana se caractérise par de vastes espaces verts, des déplacements très limités, une homogénéité sociale et un sentiment prononcé de sécurité. Remarquons toutefois que, la plupart du temps, Fontana a été découvert par hasard en tant que lieu résidentiel, après de multiples investigations ou par le bouche à oreille. Pour pouvoir y accéder, quelques familles sont allées jusqu'aux limites extrêmes de leurs possibilités financières. Le cliché selon lequel Fontana est destiné exclusivement aux "super riches » est donc inexact; dans certains cas, l'équipement intérieur du logement demeure très simple et/ou inachevé, parfois durant des années. La plupart des familles interrogées ont habité préalablement dans l'espace urbain avant de devenir des résidents suburbains, en totale conformité avec le modèle courant du cycle de vie (cf. Häussermann/

Siebel, 2004, p. 159), généralement jusqu'à la première grossesse de la maman. La proximité de la ville-centre joue à ce sujet un rôle très différencié. D'une part, la plupart des lieux de travail sont localisés en ville. Par contre, mères et enfants vivent sur place à Oberwaltersdorf et dans les communes avoisinantes, où les besoins d'approvisionnement quotidiens de même qu'une offre satisfaisante en établissements d'enseignement secondaire sont garantis. La « ville » est souvent perçue comme un lieu auquel on ne veut plus retourner pour diverses raisons : la crainte de la criminalité, des nuisances atmosphériques et de celles causées par le trafic, la crainte aussi de l'anonymat.

On attache ainsi beaucoup d'importance à la qualité des relations de voisinage, aux contacts suivis, par enfants interposés fréquentant le jardin d'enfants ou l'école primaire d'Oberwaltersdorf, avec d'autres jeunes familles de « Fontana », voire, entretemps, avec des familles de la commune d'Oberwaltersdorf. Parallèlement à la satisfaction de bénéficier d'une qualité résidentielle de premier ordre, les résidents sont heureux de faire partie de la " communauté ", forgée progressivement à l'intérieur de Fontana, grâce à laquelle il est possible de bénéficier de rencontres régulières (Soirées "grill» au "Clubhouse », au Club du cigare,...). Cette ambiance conviviale est également sous-tendue par un sentiment, très subjectif, de sécurité et de protection face aux agressions et nuisances « extérieures ».

Cependant, dans ce contexte, les réponses divergent déjà nettement, dès lors qu'est évoquée la question de la transformation du Parc en "gated community ». Tandis que quelques résidents abonderaient dans ce sens et seraient prêts à en payer le prix financier, d'autres récusent catégoriquement un tel projet, dans la mesure où ils ne souhaitent pas que leurs enfants soient privés, entièrement ou partiellement, du contact avec ceux de leur âge, y compris en-dehors de Fontana.

\section{B. Fontana, une nouvelle qualité de suburbanisation résidentielle}

Les spécificités du concept de Fontana et, par là-même, les différences avec les projets usuels de lotissements et le processus de réalisations résidentielles en milieu suburbain apparaissent nettement: dans le cas de Fontana en l'occurrence, un investisseur central acquiert une aire sur laquelle la commune ne décide plus ni des conditions de l'aménagement du bâti ni de la structure d'ensemble des constructions. En tant que lotisseur privé, c'est lui qui est le seul décideur. De plus, l'homogénéité et une forte 
image confèrent à l'ensemble une plus-value identitaire élevée, ce qui contraste avec la situation de la plupart des lotissements suburbains, y compris ceux prévus pour les couches aisées de la population, construits depuis les années cinquante jusqu'au début des années soixante. Fontana se singularise notamment par une uniformité intérieure très consciente, très appréciée par beaucoup de résidents; l'ensemble tranche nettement avec les structures banales de lotissements suburbains et crée ce que l'on pourrait appeler une "adresse » impossible à confondre avec une autre réalisation, grâce à la combinaison d'éléments imagés, spatiaux et associatifs uniques (Bölling, 2004, p. 98). Grâce à leurs avantages économiques et sociaux se situant essentiellement au-dessus de la moyenne des possibilités des autres types de population, les habitants de Fontana bénéficient d'un lieu résidentiel qui correspond à leurs préférences et à leurs aspirations.

Quoique les motivations fondamentales des résidents de Fontana correspondent en général à celles de tous ceux qui accordent leur préférence à la suburbanisation traditionnelle, elles se distinguent entièrement de celle-ci au point de vue qualitatif, une qualité toute nouvelle. Se pose cependant la question de savoir dans quelle mesure ce modèle est susceptible d'intéresser dans un futur proche une clientèle suffisamment élargie pour favoriser son extension à de nouvelles aires suburbaines.

\section{Conclusion : la région urbaine de Vienne a atteint la phase postsuburbaine}

L'exemple de la région urbaine de Vienne permet de constater aisément que les tendances de développement postsuburbaines ne doivent pas nécessairement être précédées d'une phase de suburbanisation quantitativement importante. La mutation qualitative est surtout conditionnée par la nouvelle donne économique à l'échelle internationale et la transformation des perceptions et préférences de la population.

La commune de Korneuburg constitue ainsi un modèle dans ce domaine, étant parvenue à se hisser au niveau d'une localité hautement attractive grâce à une vaste offre innovative, pour s'affirmer en tant que pôle économique autonome à l'intérieur de la région urbaine. Compte tenu des tendances internationales privilégiant des lieux d'activités multifonctionnels, de même que l'implantation d'établissements d'enseignement supérieur privé et d'autres équipements orientés vers les hautes technologies, tels que ceux relatifs à la recherche-développement, dite R\&D (BORSDORF 2004, p. 22), les prévisions de l'administration de Korneuburg peuvent être couronnées de succès; cette commune est susceptible de constituer un lieu attractif permanent dans la région urbaine de Vienne.

Le développement de la ville de Mödling montre que les habitants de communes postsuburbaines deviennent lentement conscients des qualités (encore cachées) de leur lieu d'habitation, qualités qu'ils cherchent à rentabiliser de plusieurs façons: de nouveaux styles de vie ou des concepts d'affaires d'une part, un engagement citoyen renforcé en faveur du développement urbain sont les caractéristiques d'une mutation significative d'une ville-dortoir en commune consciente des atouts de son devenir (Borsdorf/Mayer, 2003, p. 5).

51 Le parc résidentiel Fontana montre que des tendances urbaines ségrégationnistes sont à même de gagner en qualité dans un espace postsuburbain, quoique les pratiques des 
«Häuslebauer» (constructeurs de maisons sans cachet) n'aient pas connu de modification sensible depuis le début de la suburbanisation.

Les profils des trois communes de la région de Vienne apparaissent donc comme significatives du vaste processus de postsuburbanisation. Les communes affinent et affirment de plus en plus leur profil qualitatif et leur identité; leur spécificité fonctionnelle progresse constamment et pèse d'un poids certain dans la transformation de l'organisation économique, infrastructurelle et socio-culturelle de la région urbaine. Quoique le phénomène de postsuburbanisation évolue selon des modèles analogues à l'échelle internationale, son application originale dans la région urbaine de Vienne mérite une attention particulière.

\section{BIBLIOGRAPHIE}

ARING J. \& HERFERT G. (2001). — « Neue Muster der Wohnsuburbanisierung. » In : BRAKE K. et al. (sous la direction de), Suburbanisierung in Deutschland. Aktuelle Tendenzen, Opladen, pp. 43-56.

BAUER-WOLF S. (2003). - Stadt-Umland-Migration Wien. Erforschung zielgruppenspezifischer Interventionspotenziale. Endbericht, Wien, Stadt Wien MA 50.

BORSDORF A. (2004). - « On the way to Post-Suburbia ? Changing structures in the outskirts of European cities ». In : BORSDORF A. \& ZEMBRI P. (sous la direction de), European Cities - Insights on Outskirts. Structures, COST Action C10, Brussels, Blanchard, pp. 7-30.

BORSDORF A. \& MAYER V. (2003). - « Konvergenz und Divergenz der Kulturen in den Randzonen der Städte. Eine notwendige Einleitung ». TRANS. Internetzeitschrift für Kulturwissenschaften, 15 http:// www.inst.at/trans/15Nr/03_7/borsdorf_mayer15.htm, 24 auguste 2005.

BöLLING L. (2004). - « Zwischenstadt lesen. Spurensuche zwischen ,Downtown EschbornSossenheim' und ,Airport-City Rhein-Main' ». In : BöLLING L. \& SIEVERTS T. (sous la direction de), Mitten am Rand. Auf dem Weg von der Vorstadt über die Zwischenstadt zur regionalen Stadtlandschaft, Wuppertal, pp. 94-113.

BRAKE K. (2001). - « Neue Akzente der Suburbanisierung. Suburbaner Raum und Kernstadt : Eigene Profile und neuer Verbund ». In : BRAKE K. et al. (sous la direction de), Suburbanisierung in Deutschland. Aktuelle Tendenzen, Opladen, pp. 15-26.

EINACKER I. \& MÄDING H. (2004). - « Kräfte und Akteure in der Zwischenstadt ». In : BÖLLING L. \& SIEVERTS T. (sous la direction de), Mitten am Rand. Auf dem Weg von der Vorstadt über die Zwischenstadt zur regionalen Stadtlandschaft, Wuppertal, pp. 186-189.

FASSMANN H. \& HATZ G. (2004). - « Vier Viertel und ein paar Städte - eine regionalgeographische Skizze ». In : WAGNER M. (sous la direction de), Kulturgeschichte Niederösterreichs, Band 1, Wien, pp. 165-191.

HATZ G. (2002). - « Wien im Umbruch. Aktuelle Prozesse der Stadtentwicklung », Geographische Rundschau 9, pp. 4-9.

HÄUSSERMANN H. \& SIEBEL W. (2004). - Stadtsoziologie. Eine Einführung, Frankfurt. 
KAGERMEIER A.et al. (2001). - « Die Region München. Auf dem Weg zu regionalen

Patchworkstrukturen ». In : BRAKE K.et al.(sous la direction de), Suburbanisierung in Deutschland.

Aktuelle Tendenzen, Opladen, pp. 163-174.

KULKE E. (2001). - « Entwicklungstendenzen suburbaner Einzelhandelslandschaften ». In : BRAKE K. et al. (sous la direction de), Suburbanisierung in Deutschland. Aktuelle Tendenzen, Opladen, pp. 57-70.

MARIK S. (2002). - « Das Wiener Stadtumland ». In : FASSMANN H. \& HATZ G. (sous la direction de), Wien. Stadtgeographische Exkursionen, Wien, pp. 283-303.

MAYER V. (2004). - Wohnbauentwicklung und Wohnqualität im Umland von Wien. Ergebnisse einer Bürgermeisterbefragung, Wien, Eigenverlag Akademie der Wissenschaften, 181 p.

ÖSTERREICHISCHE RAUMORDNUNGSKONFERENZ ÖROK (2005). - ÖROK Prognosen 2001-2031. Teil 2 : Haushalte und Wohnungsbedarf nach Regionen und Bezirken Österreichs, Wien.

BÜRO PAULA (2002). - Stadtteilplanung Werft/Exerzierplatz. Endbericht, Wien, inédit.

Internet, information additionnelle :

http://www.gpl.at

http://immofonds.korneuburg.gv.at

www.bluedanubepark.co.at

http://www.moedling.at

http://www.stadtmarketing-moedling.at

\section{NOTES}

1. L'Institut de géographie et de recherche régionale de l'Université de Vienne est en train de réaliser une étude portant sur une année consacrée à L'américanisation de la « suburbia » viennoise? L'exemple du parc résidentiel Fontana. Les indications livrées dans la présente contribution sont fondées sur des entretiens réalisés avec les responsables de l'administration gestionnaire de Fontana, ainsi qu'avec des résidents concernés. Fontana n'a rien d'exceptionnel pour les standards américains. En Europe cependant, cette implantation continue à constituer un phénomène peu ordinaire. La demande de terrains à bâtir se poursuit de manière régulière. Se pose alors la question de connaître le profil des clients.

\section{RÉSUMÉS}

Du point de vue quantitatif, la suburbanisation dans la région urbaine de Vienne n'a occupé qu'une place très modeste au cours des dernières décennies. Comparées aux villes allemandes, la diminution de la population en centre-ville tout autant que l'explosion urbaine en périphérie n'ont pas été aussi importantes dans la région urbaine de Vienne. En ce qui concerne toutefois le développement suburbain, celui-ci présente des exemples typiques dans l'ensemble de la région urbaine, à la fois dans les aires qui ont été suburbanisées en premier et dans la partie de la 
périphérie à dominante rurale.

Pour faire ressortir la grande variété des caractéristiques socio-économiques, culturelles et architecturales, susceptibles d'être partie prenante de l'idée de "postsuburbia ", nous avons choisi de présenter dans cet article l'évolution de trois communes de la région urbaine viennoise vers la " postsuburbia ». Au départ, les profils de base de ces trois communes sont très différents les uns des autres :

à la suite de la fermeture d'un "dockyard», la municipalité de Korneuburg a procédé courageusement à l'indispensable restructuration; aussi est-elle en train de réaliser sur un terrain en friche un concept attractif associant un parc technologique, des institutions culturelles et bien d'autres équipements. Parallèlement, Mödling peut être considérée comme une «Belle au bois dormant ", une ville qui a été plus ou moins submergée par la suburbanisation il y a quelques décennies, et dont on peut désormais observer une certaine relance: l'administration et les habitants ont pris conscience des potentialités et des atouts de cette ville ; par une démarche volontariste de développement, ils visent une place de choix en ce qui concerne des commerces de haut niveau, ainsi que la croissance de structures diverses. Le «Fontana Park» est une combinaison de financements privés en vue de réalisations résidentielles pour les couches supérieures de la population, de l'aménagement d'un golf et d'un quartier général européen pour une firme mondiale. A bien des égards, ce projet se caractérise par l'émergence d'une nouvelle qualité de suburbanisation résidentielle.

Post-suburban structures in the city region of Vienna. - From a quantitative point of view suburbanisation in Vienna region took place very moderately within the last decades. Compared to german cities both the decline of population within in the core city and the urban sprawl in the surrounding area are less distinctive. Nevertheless: concerning postsuburban development some striking examples can be found in the whole city region, in early suburbanized areas as well as in the still predominant rural part of the periphery.

To show the great variety of socio-economic, cultural or architectural characteristics that can be subsumed under the idea of postsuburbia, the way to postsuburbia of three communes in Vienna city region will be outlined in the article. Their basic conditions are very different: As a consequence of the closure of a dockyard the municipality of Korneuburg is courageously facing the needful restructuring and is on the way to realize an attractive concept on the former wasteland including a technology park, cultural institutions and much more. At the same time Mödling can be seen as a "Sleeping Beauty" - a town that was more or less overwhelmed by suburbanization some decades ago and where now a certain kind of relaunch can be observed: both administration and inhabitants became aware of the potential and qualities of the town and are forcing its development towards the first place to be concerning upscale shopping and living within grown structures in different ways. "The Fontana" - Park is a privately financed combination of upper-class residential estate, golf course and European headquarter of a worldwide company. In many aspects this project marks the start of a new quality of residential suburbanization.

Post-suburbane Strukturen im Verdichtungsraum Wien. - Aus rein quantitativer Sicht ist der Suburbanisationsprozess in Wien in den letzten Dekaden sehr moderat verlaufen. Im Vergleich zu deutschen Städten sind sowohl der Bevölkerungsverlust des Stadtkerns als auch der « urban sprawl » in die Randzonen weniger stark ausgeprägt. Dennoch können hinsichtlich der neuen Tendenzen post-suburbaner Entwicklungen einige Beispiele im Wiener Raum aufgezeigt werden. Sie finden sich sowohl in den früh suburbanisierten Stadtarealen als auch in den noch immer weitgehend ländlich strukturierten Räumen der Peripherie.

In diesem Beitrag werden drei Gemeinden der Stadtregion Wien vorgestellt, in denen sehr unterschiedliche Entwicklungen stattgefunden haben. In Korneuburg ist es die Umnutzung eines ehemaligen Industriestandorts, die neue Impulse für Technologieentwicklung, Kultur und 
manches andere freisetzt. In Mödling, einer Randgemeinde, die noch vor kurzem in Gefahr war, zur Gänze «suburbanisiert » zu werden, werden Bürger und Stadtverwaltung zunehmend des Potentials für eine eigene Entwicklung bewusst, wobei hochwertige Konsum - und Wohninfrastrukturen geschaffen werden. Der Fontana-Park schließlich ist eine nordamerikanischen Gated Communities nur im weitesten Sinn vergleichbare Wohnzelle « auf der grünen Wiese », die in der Architektursprache der Neuen Welt exklusiven Wohnraum für gehobene Schichten bereitstellt, mit künstlichem See, Golfplatz ausgestattet ist, anders aber als die umzäunten Wohnviertel und Städte der Neuen Welt auch das « headquarter » eines global agierenden Unternehmens beherbergt. Alle drei Beispiele weisen weit über frühere Suburbanisierungsphänomene hinaus und belegen die neue Qualität der Post-Suburbanisierung.

\section{INDEX}

Mots-clés : fragmentation, géographie urbaine, postsuburbia, région urbaine, ségrégation, Vienne

Schlüsselwörter : Fragmentierung, Postsuburbia, Segregation, städtische Geographie, städtische Region, Wien

Keywords : city region, fragmentation, postsuburbia, segregation, urban geography, Vienna

\section{AUTEUR}

PETER J. GÖRGL

Institut für Geographie und Regionalforschung, Universitätsstraße 7, A- 1010 Wien 